\title{
Children and youth with non-traumatic brain injury: a population based perspective
}

\author{
Vincy Chan ${ }^{1,2,3^{*}}$, Jason D. Pole ${ }^{3,4}$, Michelle Keightley ${ }^{2,5}$, Robert E. Mann ${ }^{4,6}$ and Angela Colantonio ${ }^{1,2,4}$
}

\begin{abstract}
Background: Children and youth with non-traumatic brain injury (nTBI) are often overlooked in regard to the need for post-injury health services. This study provided population-based data on their burden on healthcare services, including data by subtypes of nTBI, to provide the foundation for future research to inform resource allocation and healthcare planning for this population.

Methods: A retrospective cohort study design was used. Children and youth with nTBI in population-based healthcare data were identified using International Classification of Diseases Version 10 codes. The rate of nTBI episodes of care, demographic and clinical characteristics, and discharge destinations from acute care and by type of nTBI were identified.

Results: The rate of pediatric nTBI episodes of care was 82.3 per 100,000 ( $N=17,977)$; the average stay in acute care was 13.4 days ( $S D=25.6$ days) and $35 \%$ were in intensive care units. Approximately $15 \%$ were transferred to another inpatient setting and $6 \%$ died in acute care. By subtypes of nTBI, the highest rates were among those with a diagnosis of toxic effect of substances (22.7 per 100,000), brain tumours (18.4 per 100,000), and meningitis (15.4 per 100,000). Clinical characteristics and discharge destinations from the acute care setting varied by subtype of $\mathrm{nTBl}$; the proportion of patients that spent at least one day in intensive care units and the proportion discharged home ranged from $25.9 \%$ to $58.2 \%$ and from $50.6 \%$ to $76.4 \%$, respectively.

Conclusions: Children and youth with nTBI currently put an increased demand on the healthcare system. Active surveillance of and in-depth research on nTBI, including subtypes of nTBI, is needed to ensure that timely, appropriate, and targeted care is available for this pediatric population.
\end{abstract}

Keywords: Non-traumatic brain injury, International Classification of Diseases, Pediatrics

\section{Background}

Acquired brain injury (ABI) is "an insult to the brain that affects its structure or function, resulting in impairments of cognition, communication, physical function, or psychosocial behavior" and "does not include brain injuries that are congenital, degenerative, or induced by birth trauma" [1]. To date, much attention has been placed on brain injuries from traumatic causes (i.e., a traumatic brain injury, TBI). However, it is important to

\footnotetext{
* Correspondence: vincy.chan@uhn.ca

${ }^{1}$ Toronto Rehabilitation Institute, University Health Network, 550 University

Avenue, Toronto, ON M5G 2A2, Canada

${ }^{2}$ Rehabilitation Sciences Institute, University of Toronto, 500 University

Avenue, Toronto, ON M5G 1 V7, Canada

Full list of author information is available at the end of the article
}

recognize that brain injuries from non-traumatic causes (i.e., non-traumatic brain injury, nTBI) can also result in negative and long-term consequences [2]. These nTBI include anoxia, vascular insults, toxic effect of substances, brain tumours, meningitis, metabolic encephalopathy, encephalitis, and other brain disorders [1].

Comprehensive information on the health service use among the nTBI population is currently only available for adult and older adult populations [3-12]. These studies found that the direct cost of healthcare services for nTBI was higher than that of TBI (\$120.7 vs. \$368.7 million) [6]. In Ontario, Canada, between the fiscal years of 2003/04 and 2009/10, approximately $10 \%$ of nTBI cases in the acute care setting were among patients aged 
18 years and under [9]. An understanding of this pediatric population is important because healthcare, in particular rehabilitation, for the pediatric nTBI population often occurs in similar or identical programs and facilities as those of TBI $[13,14]$. However as a group, nTBI are often overlooked with regard to the potential for long lasting sequelae and the need for healthcare services post-injury [15].

Equally important is the need to understand each subtype of nTBI, as nTBI includes diverse health conditions that may require a targeted approach to resource allocation and healthcare planning. Unfortunately, there is currently a lack of population based research and data on subtypes of pediatric nTBI with regard to their healthcare use. For example, even though primary brain tumours are the leading causes of cancer death in children and youth aged 19 years and under [16, 17], there lacks a general epidemiological profile and healthcare utilization information for this population. Research on childhood cancer indicate that there is no clear end to the duration of healthcare need among survivors and these patients continue to use substantial healthcare resources that are not accounted for in resource allocation [18]. As such, children and youth with brain tumors may be candidates for increased healthcare use. Similarly, a systematic review of out-of-hospital pediatric cardiac arrest and drowning, which are common causes of anoxic brain injuries $[19,20]$, showed that, while less than $7 \%$ of children with cardiac arrest survive to hospital discharge, only $2.2 \%$ survive without neurological sequelae. A fifth of patients with anoxic brain injuries due to near drowning survive to hospital discharge, but survival can be as high as $80 \%$ if timely and appropriate actions are taken [19]. This suggests that this population is also likely to be users of acute-care services and in particular, post-hospitalization services such as homecare or rehabilitation. NTBI from infectious causes (e.g., meningitis, encephalitis) are most common among pediatric age groups, with persisting sequelae often reported, including fatigue, epilepsy, and impairments in cognition, memory, and motor [21-24]. As such, despite the diversity of causes of nTBI, the neurological sequelae and need for health services are evident across subtypes of nTBI.

The objectives of this study are to provide populationbased information on (1) the burden of nTBI on healthcare services by identifying the rate of nTBI episodes of care in the province of Ontario in Canada and (2) the demographic and clinical characteristics and discharge destinations of hospitalized children and youth aged 19 years and under with nTBI. Recognizing the diverse conditions captured as nTBI, this paper additionally aimed to provide data on subtypes of nTBI that can be used to guide additional research on each type of nTBI. An understanding of the epidemiological profile and healthcare use of this group of individuals can greatly assist to appropriately, adequately, and effectively plan healthcare services for this population to ensure that their needs are met. This study is a first step towards a greater understanding of children and youth with nTBI and the patterns of their healthcare use.

\section{Methods}

The Canadian Institute for Health Information (CIHI) National Ambulatory Care Reporting System (NACRS) and the Discharge Abstract Database (DAD) were used. The NACRS is a mandated data collection system that collects emergency department (ED) and ambulatory care data [25]. A reabstraction study of the NACRS data that compared 7,500 charts from 15 hospitals in Ontario from 2004 to 2005 indicated up to $80 \%$ agreement for International Classification of Diseases Version 10 (ICD10) codes for patients' main problem (i.e., the health condition responsible for the patient's visit, requiring evaluation and/or treatment/management) [25]. The DAD contains demographic and clinical information on all hospital admissions and discharges, including transfers and deaths, using standard diagnosis and procedure/intervention codes [26]. A reabstraction study of the DAD found high sensitivity and near perfect specificity for demographic variables and moderate to substantial agreement for diagnoses (kappa value 0.41 to 0.80) [27]. As residents of Ontario have universal access to ED and hospital-based care, these data sources allow for the identification of all children and youth with a nTBI diagnoses in the ED and/or acute care setting during our study period.

NTBI was categorized by the presence of specified ICD-10 codes in any of the 10 diagnosis fields in the NACRS and the 25 diagnosis fields in the DAD. These

Table 1 International Classification of Diseases version 10 (ICD-10) case definitions for non-traumatic brain injury

\begin{tabular}{ll}
\hline Type of Non-Traumatic brain injury & ICD-10 Codes \\
\hline Toxic effect of substances & T40.5, T42.6, T51, T56, T57.0, T57.2, \\
& T57.3, T58, T64, T65.0 \\
Anoxia & G93.1, T71, T75.1, R09.0 \\
Vascular insults (not captured in & 162.0, 162.9 \\
Other national studies of stroke) & \\
Brain tumours & C70, C71, C79.3, C79.4, D32.0, D33.0, \\
& D33.1, D33.2, D33.3, D42.0, D43, \\
& D43.2 \\
Encephalitis & A81.1, A83.0, A83.2, A86.0, B00.4, \\
& B01.1, B02.0, B05.0, B94.1, G04.0, \\
& G04.2, G04.8, G04.9, G05, G09 \\
Metabolic encephalopathy & E10.0, E11.0, E13.0, E14.0, E15, G92, \\
& G93.4 \\
Meningitis & A87, B01.0, B37.5, G00, G01, G02, \\
& G03 \\
Other brain disorders & G91.0, G91.1, G91.2, G93.2, G93.5, \\
& G93.6, G93.8, G93.9, G99.8, R29.1 \\
\hline
\end{tabular}




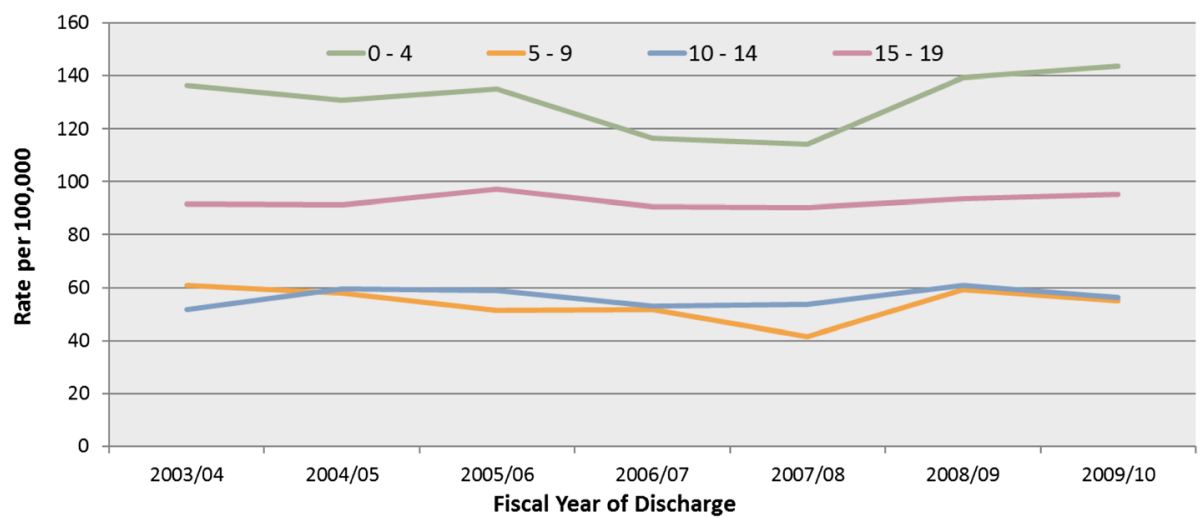

Fig. 1 nTBl episodes of care by age groups and fiscal year of discharge

ICD-10 codes represent conditions that are captured as a nTBI as defined by the Commission on Accreditation of Rehabilitation Facilities (CARF) International [1] and through stakeholder consultation in Ontario, Canada. This included members from the Ontario Ministry of Health and Long-Term Care, Ontario Agency for Protection and Promotion, SMARTRISK, and the Ministry of Transportation to advise on the creation of a neurotrauma surveillance system to inform prevention in the province of Ontario [28] (Table 1). Stroke captured in national studies were excluded from our case definition for vascular insults to reflect current research and clinical practice in Ontario, Canada. For example, various national rehabilitation information systems $[29,30]$ and centres [31,32] classify stroke separately from nTBI and some definitions of nTBI include vascular conditions that are not captured in major stroke studies [33]. As such, these vascular insults were included in this study. All subtypes of nTBI across the 10 diagnosis fields in the NACRS and 25 diagnosis fields in the DAD were counted.

Demographic variables included age and sex. Children and youth aged 19 years and under were categorized into five-year age groups [0 to 4 years (infants), 5 to 9 years (children), 10 to 14 years (youth), and 15 to 19 years (adolescents)] consistent with categories commonly used in the ABI literature [34], Statistics Canada [35], and the World Health Organization [36].

Clinical variables included the Charlson Comorbidity Index, length of stay (LOS) in acute care, and special care days. The Charlson Comorbidity Index is widely accepted as a useful tool for measuring comorbidity disease status, has been shown to have a consistent

Table 2 nTBl episodes of care (n and rate per 100,000 children and youth aged 19 years and under) in Ontario between fiscal years 2003/04 and 2009/10 by age, fiscal year of discharge, and sex

\begin{tabular}{|c|c|c|c|c|c|c|c|c|c|c|c|c|c|c|c|}
\hline & \multicolumn{3}{|l|}{ Overall } & \multicolumn{3}{|l|}{$0-4$} & \multicolumn{3}{|l|}{$5-9$} & \multicolumn{3}{|l|}{$10-14$} & \multicolumn{3}{|l|}{$15-19$} \\
\hline & $\begin{array}{l}\text { Overall } \\
\mathrm{n} \\
\text { rate }\end{array}$ & $\begin{array}{l}\text { Male } \\
\mathrm{n} \\
\text { rate }\end{array}$ & $\begin{array}{l}\text { Female } \\
\mathrm{n} \\
\text { rate }\end{array}$ & $\begin{array}{l}\text { Overall } \\
\mathrm{n} \\
\text { rate }\end{array}$ & $\begin{array}{l}\text { Male } \\
\mathrm{n} \\
\text { rate }\end{array}$ & $\begin{array}{l}\text { Female } \\
\mathrm{n} \\
\text { rate }\end{array}$ & $\begin{array}{l}\text { Overall } \\
\mathrm{n} \\
\text { rate }\end{array}$ & $\begin{array}{l}\text { Male } \\
\text { n } \\
\text { rate }\end{array}$ & $\begin{array}{l}\text { Female } \\
\mathrm{n} \\
\text { rate }\end{array}$ & $\begin{array}{l}\text { Overall } \\
\mathrm{n} \\
\text { rate }\end{array}$ & $\begin{array}{l}\text { Male } \\
\mathrm{n} \\
\text { rate }\end{array}$ & $\begin{array}{l}\text { Female } \\
\mathrm{n} \\
\text { rate }\end{array}$ & $\begin{array}{l}\text { Overall } \\
\mathrm{n} \\
\text { rate }\end{array}$ & $\begin{array}{l}\text { Male } \\
\mathrm{n} \\
\text { rate }\end{array}$ & $\begin{array}{l}\text { Female } \\
\mathrm{n} \\
\text { rate }\end{array}$ \\
\hline 2003/04 & $\begin{array}{l}2591 \\
82.7\end{array}$ & $\begin{array}{l}1437 \\
89.7\end{array}$ & $\begin{array}{l}1154 \\
75.5\end{array}$ & $\begin{array}{l}932 \\
136.3\end{array}$ & $\begin{array}{l}553 \\
158.4\end{array}$ & $\begin{array}{l}379 \\
113.3\end{array}$ & $\begin{array}{l}477 \\
61.0\end{array}$ & $\begin{array}{l}242 \\
60.7\end{array}$ & $\begin{array}{l}235 \\
61.3\end{array}$ & $\begin{array}{l}432 \\
51.6\end{array}$ & $\begin{array}{l}252 \\
58.9\end{array}$ & $\begin{array}{l}171 \\
41.8\end{array}$ & $\begin{array}{l}759 \\
91.6\end{array}$ & $\begin{array}{l}390 \\
91.5\end{array}$ & $\begin{array}{l}369 \\
91.6\end{array}$ \\
\hline 2004/05 & $\begin{array}{l}2606 \\
83.2\end{array}$ & $\begin{array}{l}1406 \\
87.7\end{array}$ & $\begin{array}{l}1200 \\
78.5\end{array}$ & $\begin{array}{l}893 \\
131.0\end{array}$ & $\begin{array}{l}522 \\
149.4\end{array}$ & $\begin{array}{l}371 \\
111.7\end{array}$ & $\begin{array}{l}445 \\
58.0\end{array}$ & $\begin{array}{l}247 \\
63.2\end{array}$ & $\begin{array}{l}198 \\
52.7\end{array}$ & $\begin{array}{l}500 \\
59.4\end{array}$ & $\begin{array}{l}267 \\
62.1\end{array}$ & $\begin{array}{l}233 \\
56.6\end{array}$ & $\begin{array}{l}768 \\
91.3\end{array}$ & $\begin{array}{l}370 \\
85.5\end{array}$ & $\begin{array}{l}398 \\
97.3\end{array}$ \\
\hline 2005/06 & $\begin{array}{l}2635 \\
84.2\end{array}$ & $\begin{array}{l}1455 \\
90.8\end{array}$ & $\begin{array}{l}1180 \\
77.3\end{array}$ & $\begin{array}{l}922 \\
135.1\end{array}$ & $\begin{array}{l}539 \\
153.8\end{array}$ & $\begin{array}{l}383 \\
115.4\end{array}$ & $\begin{array}{l}385 \\
51.4\end{array}$ & $\begin{array}{l}226 \\
59.1\end{array}$ & $\begin{array}{l}159 \\
43.3\end{array}$ & $\begin{array}{l}494 \\
58.9\end{array}$ & $\begin{array}{l}278 \\
64.9\end{array}$ & $\begin{array}{l}216 \\
52.6\end{array}$ & $\begin{array}{l}834 \\
97.2\end{array}$ & $\begin{array}{l}412 \\
93.5\end{array}$ & $\begin{array}{l}422 \\
101.1\end{array}$ \\
\hline 2006/07 & $\begin{array}{l}2419 \\
77.3\end{array}$ & $\begin{array}{l}1327 \\
82.8\end{array}$ & $\begin{array}{l}1092 \\
71.6\end{array}$ & $\begin{array}{l}808 \\
116.4\end{array}$ & $\begin{array}{l}441 \\
123.6\end{array}$ & $\begin{array}{l}367 \\
108.8\end{array}$ & $\begin{array}{l}379 \\
51.8\end{array}$ & $\begin{array}{l}242 \\
64.6\end{array}$ & $\begin{array}{l}137 \\
38.4\end{array}$ & $\begin{array}{l}440 \\
53.0\end{array}$ & $\begin{array}{l}228 \\
53.9\end{array}$ & $\begin{array}{l}212 \\
52.2\end{array}$ & $\begin{array}{l}792 \\
90.7\end{array}$ & $\begin{array}{l}416 \\
92.9\end{array}$ & $\begin{array}{l}376 \\
88.4\end{array}$ \\
\hline 2007/08 & $\begin{array}{l}2328 \\
74.7\end{array}$ & $\begin{array}{l}1269 \\
79.5\end{array}$ & $\begin{array}{l}1059 \\
69.7\end{array}$ & $\begin{array}{l}794 \\
114.1\end{array}$ & $\begin{array}{l}433 \\
120.9\end{array}$ & $\begin{array}{l}361 \\
106.8\end{array}$ & $\begin{array}{l}300 \\
41.6\end{array}$ & $\begin{array}{l}176 \\
47.5\end{array}$ & $\begin{array}{l}124 \\
35.3\end{array}$ & $\begin{array}{l}439 \\
53.8\end{array}$ & $\begin{array}{l}233 \\
55.9\end{array}$ & $\begin{array}{l}206 \\
51.6\end{array}$ & $\begin{array}{l}795 \\
90.3\end{array}$ & $\begin{array}{l}427 \\
94.8\end{array}$ & $\begin{array}{l}368 \\
85.6\end{array}$ \\
\hline 2008/09 & $\begin{array}{l}2717 \\
87.5\end{array}$ & $\begin{array}{l}1453 \\
91.3\end{array}$ & $\begin{array}{l}1264 \\
83.4\end{array}$ & $\begin{array}{l}975 \\
139.5\end{array}$ & $\begin{array}{l}578 \\
160.8\end{array}$ & $\begin{array}{l}397 \\
116.9\end{array}$ & $\begin{array}{l}424 \\
59.1\end{array}$ & $\begin{array}{l}240 \\
65.2\end{array}$ & $\begin{array}{l}184 \\
52.7\end{array}$ & $\begin{array}{l}488 \\
60.8\end{array}$ & $\begin{array}{l}245 \\
59.8\end{array}$ & $\begin{array}{l}243 \\
62.0\end{array}$ & $\begin{array}{l}830 \\
93.6\end{array}$ & $\begin{array}{l}390 \\
86.1\end{array}$ & $\begin{array}{l}440 \\
101.4\end{array}$ \\
\hline 2009/10 & $\begin{array}{l}2681 \\
86.3\end{array}$ & $\begin{array}{l}1404 \\
88.3\end{array}$ & $\begin{array}{l}1277 \\
84.2\end{array}$ & $\begin{array}{l}986 \\
143.5\end{array}$ & $\begin{array}{l}561 \\
159.1\end{array}$ & $\begin{array}{l}425 \\
127.1\end{array}$ & $\begin{array}{l}399 \\
55.1\end{array}$ & $\begin{array}{l}228 \\
61.6\end{array}$ & $\begin{array}{l}171 \\
48.3\end{array}$ & $\begin{array}{l}459 \\
56.2\end{array}$ & $\begin{array}{l}218 \\
52.3\end{array}$ & $\begin{array}{l}241 \\
60.2\end{array}$ & $\begin{array}{l}837 \\
95.3\end{array}$ & $\begin{array}{l}397 \\
88.1\end{array}$ & $\begin{array}{l}440 \\
102.9\end{array}$ \\
\hline $2003 / 04-2009 / 10$ & $\begin{array}{l}17977 \\
82.3\end{array}$ & $\begin{array}{l}9751 \\
87.2\end{array}$ & $\begin{array}{l}8226 \\
77.2\end{array}$ & $\begin{array}{l}6310 \\
130.8\end{array}$ & $\begin{array}{l}3627 \\
146.5\end{array}$ & $\begin{array}{l}2683 \\
114.3\end{array}$ & $\begin{array}{l}2809 \\
54.1\end{array}$ & $\begin{array}{l}1601 \\
60.3\end{array}$ & $\begin{array}{l}1208 \\
47.6\end{array}$ & $\begin{array}{l}3243 \\
56.1\end{array}$ & $\begin{array}{l}1721 \\
58.3\end{array}$ & $\begin{array}{l}1522 \\
53.8\end{array}$ & $\begin{array}{l}5615 \\
92.9\end{array}$ & $\begin{array}{l}2802 \\
90.4\end{array}$ & $\begin{array}{l}2813 \\
95.5\end{array}$ \\
\hline
\end{tabular}


correlation to in-hospital mortality, and is used to assess the severity of comorbid health conditions [37, 38]. The score is derived using the sum of the standard weights ( 1 to 6$)$ that are assigned for each condition in the index [39]. As most patients identified in this study have few Charlson comorbidities, the categories of $0-1$ and $2+$ were used to avoid the risk of reidentification of any patients in the acute care setting. LOS in acute care was defined as the number of days between admission and discharge. Special care days were defined as the cumulative number of days spent in all intensive care units.

Table 3 Patient characteristics among children and youth with a nTBI diagnostic code in acute care in Ontario between fiscal years 2004/05 and 2009/10 by type of nTBI

\begin{tabular}{|c|c|c|c|c|c|c|c|c|c|c|c|c|c|c|c|c|c|c|}
\hline \multirow[t]{2}{*}{ Characteristics } & \multicolumn{2}{|c|}{ Overall $^{a}$} & \multicolumn{2}{|c|}{$\begin{array}{l}\text { Toxic } \\
\text { effect of } \\
\text { substances }\end{array}$} & \multicolumn{2}{|c|}{ Anoxia } & \multicolumn{2}{|c|}{$\begin{array}{l}\text { Vascular } \\
\text { insults }\end{array}$} & \multicolumn{2}{|c|}{$\begin{array}{l}\text { Brain } \\
\text { tumours }\end{array}$} & \multicolumn{2}{|c|}{ Encephalitis } & \multicolumn{2}{|c|}{$\begin{array}{l}\text { Metabolic } \\
\text { encephalopathy }\end{array}$} & \multicolumn{2}{|c|}{ Meningitis } & \multicolumn{2}{|c|}{$\begin{array}{l}\text { Other } \\
\text { brain } \\
\text { disorders }\end{array}$} \\
\hline & $N$ & $\mathrm{Col} \%$ & $N$ & Col $\%$ & $N$ & $\mathrm{Col} \%$ & $N$ & $\mathrm{Col} \%$ & $N$ & Col $\%$ & $N$ & $\mathrm{Col} \%$ & $N$ & $\mathrm{Col} \%$ & $N$ & $\mathrm{Col} \%$ & $N$ & $\mathrm{Col} \%$ \\
\hline Overall & 6102 & 100 & 713 & 100 & 903 & 100 & 182 & 100 & 745 & 100 & 438 & 100 & 325 & 100 & 2094 & 100 & 1077 & 100 \\
\hline \multicolumn{19}{|l|}{ Age Groups } \\
\hline $0-4$ & 2713 & 44.5 & 59 & 8.3 & 530 & 58.7 & 108 & 59.3 & 174 & 23.4 & 177 & 40.4 & 117 & 36.0 & 1301 & 62.1 & 387 & 35.9 \\
\hline $5-9$ & 819 & 13.4 & 13 & 1.8 & 123 & 13.6 & 18 & 9.9 & 182 & 24.4 & 98 & 22.4 & 55 & 16.9 & 225 & 10.7 & 165 & 15.3 \\
\hline $10-14$ & 872 & 15.9 & 97 & 13.6 & 99 & 11.0 & 31 & 17.0 & 190 & 25.5 & 77 & 17.6 & 70 & 21.5 & 220 & 10.5 & 249 & 23.1 \\
\hline $15-19$ & 1598 & 26.2 & 544 & 76.3 & 151 & 16.7 & 25 & 13.7 & 199 & 26.7 & 86 & 19.6 & 83 & 25.5 & 303 & 14.5 & 276 & 25.6 \\
\hline \multicolumn{19}{|l|}{ Sex } \\
\hline Males & 3358 & 55.0 & 321 & 45.0 & 539 & 59.7 & 114 & 62.6 & 410 & 55.0 & 224 & 51.1 & 172 & 52.9 & 1163 & 56.8 & 595 & 55.3 \\
\hline Females & 2744 & 45.0 & 392 & 55.0 & 364 & 40.3 & 68 & 37.4 & 335 & 45.0 & 214 & 48.9 & 153 & 47.1 & 886 & 43.2 & 482 & 44.8 \\
\hline \multicolumn{19}{|l|}{ Length of Stay (Days) } \\
\hline $\begin{array}{l}\text { Average Length of } \\
\text { Stay (Mean, SD) }\end{array}$ & \multicolumn{2}{|c|}{$13.4(25.6)$} & \multicolumn{2}{|c|}{$4.0(6.4)$} & \multicolumn{2}{|c|}{$15.2(36.4)$} & \multicolumn{2}{|c|}{$26.7(44.1)$} & \multicolumn{2}{|c|}{$14.0(22.4)$} & \multicolumn{2}{|c|}{$16.7(23.9)$} & \multicolumn{2}{|c|}{$20.2(41.0)$} & \multicolumn{2}{|c|}{$13.8(21.6)$} & \multicolumn{2}{|c|}{$16.0(25.9)$} \\
\hline $1-2$ & 1278 & 20.9 & 390 & 54.7 & 287 & 31.8 & 16 & 8.8 & 108 & 14.5 & 58 & 13.2 & 48 & 14.8 & 209 & 10.2 & 195 & 18.1 \\
\hline $3-5$ & 1835 & 30.1 & 224 & 31.4 & 234 & 25.9 & 40 & 22.0 & 195 & 26.2 & 107 & 24.4 & 73 & 22.5 & 699 & 34.1 & 326 & 30.3 \\
\hline $6-11$ & 1296 & 21.2 & 61 & 8.6 & 153 & 16.9 & 42 & 23.1 & 217 & 29.1 & 109 & 24.9 & 78 & 24.0 & 482 & 23.5 & 212 & 19.7 \\
\hline $12+$ & 1693 & 27.8 & 38 & 5.3 & 229 & 25.4 & 84 & 46.2 & 225 & 30.2 & 164 & 37.4 & 126 & 38.8 & 659 & 32.2 & 344 & 31.9 \\
\hline \multicolumn{19}{|c|}{ Charlson Comorbidity Index Score } \\
\hline $0-1$ & 5355 & 87.8 & $N R$ & . & 855 & 94.7 & 143 & 78.6 & 241 & 32.4 & 411 & 93.8 & 278 & 85.5 & 2005 & 97.9 & 908 & 84.3 \\
\hline $2+$ & 747 & 12.2 & $<5$ & . & 48 & 5.3 & 39 & 21.4 & 504 & 67.7 & 27 & 6.2 & 47 & 14.5 & 44 & 2.1 & 169 & 15.7 \\
\hline Special Care Days & & & & & & & & & & & & & & & & & & \\
\hline $\begin{array}{l}\text { Average Number of } \\
\text { Days (Mean, SD) }\end{array}$ & 12.5 & 22.9) & 1.9 & 2.4) & 14.6 & $(28.8)$ & 16.1 & $(22.5)$ & 4.7( & 12.8) & 13.7 & $(18.0)$ & 10.2 & 5.0) & 21.6 & (27.8) & 11.4 & (20.4) \\
\hline None & 3970 & 65.1 & 475 & 66.6 & 522 & 57.8 & 76 & 41.8 & 377 & 50.6 & 327 & 74.7 & 181 & 55.7 & 1519 & 74.1 & 591 & 54.9 \\
\hline $1-2$ & 865 & 14.2 & 197 & 27.6 & 117 & 13.0 & 21 & 11.5 & 247 & 33.2 & 25 & 5.7 & 35 & 10.8 & 114 & 5.6 & 185 & 17.2 \\
\hline $3-5$ & 370 & 6.1 & 36 & 5.1 & 70 & 7.8 & 19 & 10.4 & 69 & 9.3 & 25 & 5.7 & 43 & 13.2 & 70 & 3.4 & 94 & 8.7 \\
\hline $6-11$ & 315 & 5.2 & $<5$ & . & 70 & 7.8 & $N R$ & . & 22 & 3.0 & 20 & 4.6 & 34 & 10.5 & 97 & 4.7 & 81 & 7.5 \\
\hline $12+$ & 582 & 9.5 & $<5$ & . & 124 & 13.7 & NR & . & 30 & 4.0 & 41 & 9.4 & 32 & 9.9 & 249 & 12.2 & 126 & 11.7 \\
\hline Discharge Disposition & & & & & & & & & & & & & & & & & & \\
\hline Home & 4175 & 68.4 & 545 & 76.4 & 554 & 61.4 & 92 & 50.6 & 487 & 65.4 & 259 & 59.1 & 206 & 63.4 & 1490 & 72.7 & 653 & 60.6 \\
\hline Home with Support & 544 & 8.9 & 11 & 1.5 & 73 & 8.1 & 35 & 19.2 & 132 & 17.7 & 71 & 16.2 & 45 & 13.9 & 131 & 6.4 & 121 & 11.2 \\
\hline Rehabilitation & 47 & 0.8 & $<5$ & . & 9 & 1.0 & $<5$ & . & 9 & 1.2 & 8 & 1.8 & $<5$ & . & $<5$ & . & 15 & 1.4 \\
\hline $\begin{array}{l}\text { Complex Continuing } \\
\text { Care or Long Term Care }\end{array}$ & 81 & 1.3 & 0 & 0 & 14 & 1.6 & 7 & 3.9 & 13 & 1.7 & 15 & 3.4 & $N R$ & . & $N R$ & . & 32 & 2.9 \\
\hline Transferred & 870 & 14.3 & 151 & 21.2 & 78 & 8.6 & 24 & 13.2 & 80 & 10.7 & 76 & 17.4 & 28 & 8.6 & 372 & 18.2 & 99 & 9.2 \\
\hline Death & 385 & 6.3 & $<5$ & . & 175 & 19.4 & NR & . & 24 & 3.2 & 9 & 2.1 & 32 & 9.9 & 44 & 2.2 & 157 & 14.6 \\
\hline
\end{tabular}

Note: ${ }^{a}$ All multiple $\mathrm{nTBI}$ diagnoses were counted for and as such, the overall $\mathrm{N}$ will not add up $\mathrm{NR}=$ not reportable due to small cell sizes 
Discharge disposition from acute care included death in acute care, home, home with support services (e.g., homecare, home making supportive housing), inpatient rehabilitation, complex continuing care (CCC; e.g., chronic care facility), long term care (LTC; e.g., nursing home), and transferred to another inpatient setting.

NTBI episodes of care between fiscal years 2003/04 and $2009 / 10$ were used to determine the number and rate of healthcare utilization, which has been shown to provide a more accurate description of the utilization of healthcare services compared to data on just hospitalizations [40]. This was accomplished by linking the DAD to the NACRS via an unique encoded identifier, which is complete for all cases in the NACRS and the DAD and ensured that each episode was only captured once. The number of nTBI episodes of care by age group, sex, and type of nTBI were identified. The rate of nTBI episodes of care per 100,000 children and youth in Ontario, Canada, was calculated by dividing the total number of nTBI episodes by the population counts for the specific age group and sex in Ontario, Canada during the fiscal year of study. These numbers indicate the total number of nTBI episodes of care for every 100,000 children and youth in Ontario, Canada, during the study period.

A patient-level analysis of the patient's initial hospitalization for a nTBI between 2004/05 and 2009/10 was used to examine patient and clinical characteristics, and discharge destination from acute care. This was chosen to distinguish between a readmissions profile, which may differ from the initial admission. Patients included in this analysis must be initial hospitalizations between fiscal years 2003/04 and 2009/10. A look-back window of at least one year was used to ensure that patients included were the initial hospitalization record between fiscal years 2003/04 and 2009/10. Due to the lack of data to look back one year for fiscal year 2003/ 04 , this fiscal year was eliminated from the patient level analysis. This ensured that patients identified between fiscal years 2004/05 and 2009/10 were index hospitalizations during this study period.

\section{Results}

Between fiscal years 2003/04 and 2009/10, there were 17,977 nTBI episodes of care (82.3 per 100,000 children and youth 19 years and under in Ontario, Canada). Males had a higher rate $(87.2$ per 100,000$)$ compared to females (77.2 per 100,000). By age group, the highest rates were among infants aged 0 to 4 years (130.8 per $100,000)$, followed by adolescents aged 15 to 19 years (92.9 per 100,000$)$, youth aged 10 to 14 years (56.1 per 100,000), and children aged 5 to 9 years (54.1 per 100,000) (Table 2, Fig. 1).

Patient level analyses showed that there were 6,102 patients with nTBI between fiscal years 2004/05 and 2009/ 10. The average LOS in acute care was 13.4 days $(\mathrm{SD}=$ 25.6 days) and among those with at least one special care days (35\%), the average stay in intensive care units was 12.5 days $(\mathrm{SD}=22.9$ days). Approximately $12 \%$ of patients had a Charlson Comorbidity Index Score of 2 or higher. Most of the patients (77\%) were discharged home from acute care, of which $9 \%$ were discharged home with support services. Approximately $14 \%$ of patients were transferred to another inpatient setting, less than $1 \%$ to inpatient rehabilitation, $1 \%$ to CCC or LTC, and $6 \%$ died in acute care (Table 3, Fig. 2).

Across the types of nTBI diagnoses, toxic effect of substance episodes of care was found to be the highest (22.7 per 100,000), followed by brain tumour episodes of care (18.4 per 100,000), and meningitis episodes of care (15.4 per 100,000) (Table 4, Fig. 3). Patient and clinical characteristics and discharge destinations varied by type of nTBI diagnosis; $76 \%$ of individuals presenting with toxic effects of substances were adolescents, however, $86 \%$ of anoxic brain injury cases were infants (Fig. 4). More than

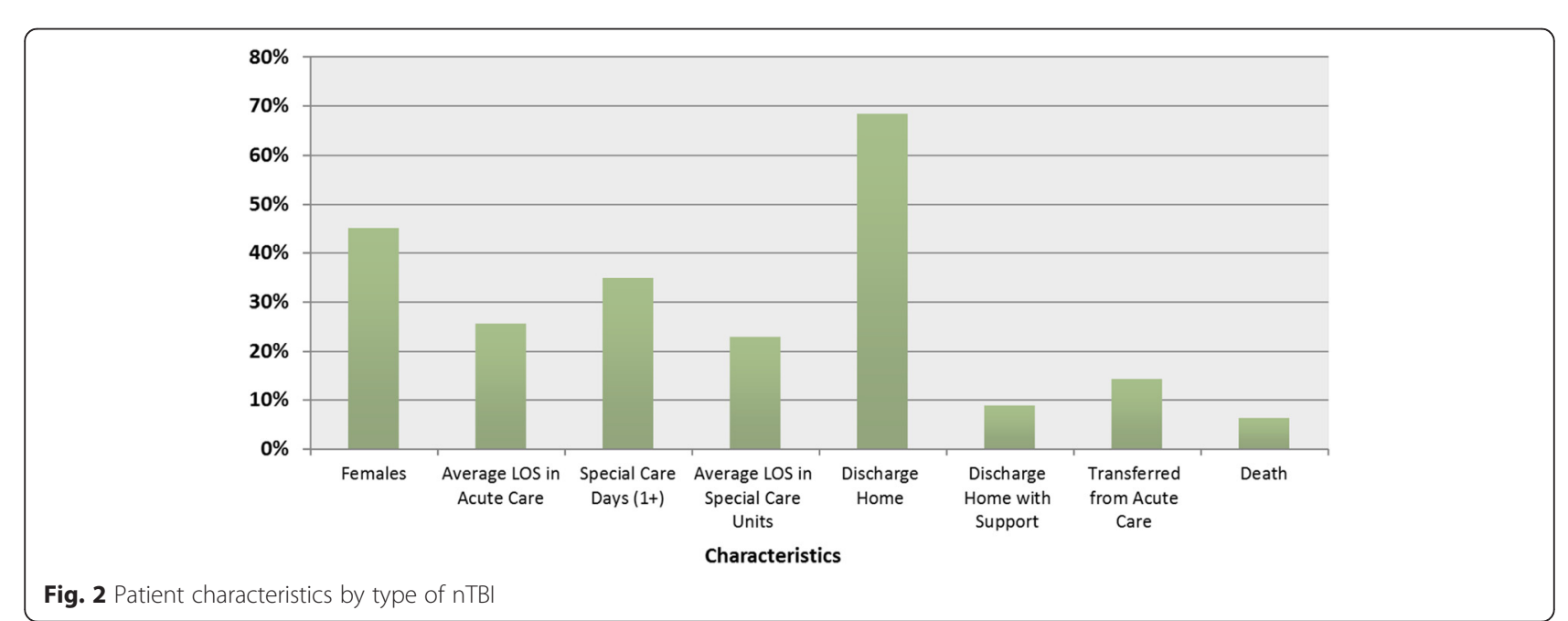


Table 4 nTBI episodes of care (n and rate per 100,000 children and youth aged 19 years and under) in Ontario between fiscal years 2003/04 and 2009/10 by type of nTBl and sex

\begin{tabular}{|c|c|c|c|c|c|c|c|c|}
\hline & $\begin{array}{l}\text { Toxic effects } \\
\text { of substance } \\
n \\
\text { rate }\end{array}$ & $\begin{array}{l}\text { Anoxia } \\
\mathrm{n} \\
\text { rate }\end{array}$ & $\begin{array}{l}\text { Vascular } \\
\text { insults } \\
\mathrm{n} \\
\text { rate }\end{array}$ & $\begin{array}{l}\text { Brain } \\
\text { tumours } \\
\mathrm{n} \\
\text { rate }\end{array}$ & $\begin{array}{l}\text { Encephalitis } \\
\mathrm{n} \\
\text { rate }\end{array}$ & $\begin{array}{l}\text { Metabolic } \\
\text { encephalopathy } \\
\mathrm{n} \\
\text { rate }\end{array}$ & $\begin{array}{l}\text { Meningitis } \\
\mathrm{n} \\
\text { rate }\end{array}$ & $\begin{array}{l}\text { Other brain } \\
\text { disorders and } \\
\text { infections } \\
\mathrm{n} \\
\text { rate }\end{array}$ \\
\hline \multicolumn{9}{|l|}{ Overall } \\
\hline 2003/04 & $\begin{array}{l}709 \\
22.6\end{array}$ & $\begin{array}{l}293 \\
9.4\end{array}$ & $\begin{array}{l}58 \\
1.9\end{array}$ & $\begin{array}{l}605 \\
19.3\end{array}$ & $\begin{array}{l}138 \\
4.4\end{array}$ & $\begin{array}{l}106 \\
3.4\end{array}$ & $\begin{array}{l}461 \\
14.7\end{array}$ & $\begin{array}{l}333 \\
10.6\end{array}$ \\
\hline 2004/05 & $\begin{array}{l}809 \\
25.8\end{array}$ & $\begin{array}{l}294 \\
9.4\end{array}$ & $\begin{array}{l}43 \\
1.4\end{array}$ & $\begin{array}{l}557 \\
17.8\end{array}$ & $\begin{array}{l}125 \\
4.0\end{array}$ & $\begin{array}{l}84 \\
2.7\end{array}$ & $\begin{array}{l}453 \\
14.5\end{array}$ & $\begin{array}{l}312 \\
10.0\end{array}$ \\
\hline 2005/06 & $\begin{array}{l}742 \\
23.7\end{array}$ & $\begin{array}{l}323 \\
10.3\end{array}$ & $\begin{array}{l}60 \\
1.9\end{array}$ & $\begin{array}{l}569 \\
18.2\end{array}$ & $\begin{array}{l}109 \\
3.5\end{array}$ & $\begin{array}{l}112 \\
3.6\end{array}$ & $\begin{array}{l}475 \\
15.2\end{array}$ & $\begin{array}{l}322 \\
10.3\end{array}$ \\
\hline 2006/07 & $\begin{array}{l}691 \\
22.1\end{array}$ & $\begin{array}{l}317 \\
10.1\end{array}$ & $\begin{array}{l}48 \\
1.5\end{array}$ & $\begin{array}{l}562 \\
18.0\end{array}$ & $\begin{array}{l}130 \\
4.2\end{array}$ & $\begin{array}{l}84 \\
2.7\end{array}$ & $\begin{array}{l}392 \\
12.5\end{array}$ & $\begin{array}{l}264 \\
8.4\end{array}$ \\
\hline 2007/08 & $\begin{array}{l}711 \\
22.8\end{array}$ & $\begin{array}{l}251 \\
8.1\end{array}$ & $\begin{array}{l}64 \\
2.1\end{array}$ & $\begin{array}{l}492 \\
15.8\end{array}$ & $\begin{array}{l}116 \\
3.7\end{array}$ & $\begin{array}{l}86 \\
2.8\end{array}$ & $\begin{array}{l}421 \\
13.5\end{array}$ & $\begin{array}{l}272 \\
8.7\end{array}$ \\
\hline 2008/09 & $\begin{array}{l}681 \\
21.9\end{array}$ & $\begin{array}{l}299 \\
9.6\end{array}$ & $\begin{array}{l}66 \\
2.1\end{array}$ & $\begin{array}{l}602 \\
19.4\end{array}$ & $\begin{array}{l}164 \\
5.3\end{array}$ & $\begin{array}{l}123 \\
4.0\end{array}$ & $\begin{array}{l}579 \\
18.6\end{array}$ & $\begin{array}{l}332 \\
10.7\end{array}$ \\
\hline 2009/10 & $\begin{array}{l}625 \\
20.1\end{array}$ & $\begin{array}{l}305 \\
9.8\end{array}$ & $\begin{array}{l}42 \\
1.4\end{array}$ & $\begin{array}{l}635 \\
20.4\end{array}$ & $\begin{array}{l}181 \\
5.8\end{array}$ & $\begin{array}{l}98 \\
3.2\end{array}$ & $\begin{array}{l}585 \\
18.8\end{array}$ & $\begin{array}{l}306 \\
9.9\end{array}$ \\
\hline $2003 / 04-2009 / 10$ & $\begin{array}{l}4968 \\
22.7\end{array}$ & $\begin{array}{l}2082 \\
9.5\end{array}$ & $\begin{array}{l}381 \\
1.7\end{array}$ & $\begin{array}{l}4022 \\
18.4\end{array}$ & $\begin{array}{l}963 \\
4.4\end{array}$ & $\begin{array}{l}693 \\
3.2\end{array}$ & $\begin{array}{l}3366 \\
15.4\end{array}$ & $\begin{array}{l}2141 \\
9.8\end{array}$ \\
\hline \multicolumn{9}{|l|}{ Males } \\
\hline 2003/04 & $\begin{array}{l}336 \\
21.0\end{array}$ & $\begin{array}{l}172 \\
10.7\end{array}$ & $\begin{array}{l}36 \\
2.2\end{array}$ & $\begin{array}{l}364 \\
22.7\end{array}$ & $\begin{array}{l}72 \\
4.5\end{array}$ & $\begin{array}{l}50 \\
3.1\end{array}$ & $\begin{array}{l}281 \\
17.5\end{array}$ & $\begin{array}{l}188 \\
11.7\end{array}$ \\
\hline 2004/05 & $\begin{array}{l}365 \\
22.8\end{array}$ & $\begin{array}{l}173 \\
10.8\end{array}$ & $\begin{array}{l}28 \\
1.7\end{array}$ & $\begin{array}{l}331 \\
20.7\end{array}$ & $\begin{array}{l}55 \\
3.4\end{array}$ & $\begin{array}{l}43 \\
2.7\end{array}$ & $\begin{array}{l}266 \\
16.6\end{array}$ & $\begin{array}{l}175 \\
10.9\end{array}$ \\
\hline 2005/06 & $\begin{array}{l}351 \\
21.9\end{array}$ & $\begin{array}{l}203 \\
12.7\end{array}$ & $\begin{array}{l}33 \\
2.1\end{array}$ & $\begin{array}{l}360 \\
22.5\end{array}$ & $\begin{array}{l}59 \\
3.7\end{array}$ & $\begin{array}{l}54 \\
3.4\end{array}$ & $\begin{array}{l}255 \\
15.9\end{array}$ & $\begin{array}{l}185 \\
11.5\end{array}$ \\
\hline 2006/07 & $\begin{array}{l}338 \\
21.1\end{array}$ & $\begin{array}{l}196 \\
12.2\end{array}$ & $\begin{array}{l}32 \\
2.0\end{array}$ & $\begin{array}{l}334 \\
20.8\end{array}$ & $\begin{array}{l}56 \\
3.5\end{array}$ & $\begin{array}{l}40 \\
2.5\end{array}$ & $\begin{array}{l}217 \\
13.5\end{array}$ & $\begin{array}{l}158 \\
9.9\end{array}$ \\
\hline 2007/08 & $\begin{array}{l}349 \\
21.9\end{array}$ & $\begin{array}{l}151 \\
9.5\end{array}$ & $\begin{array}{l}41 \\
2.6\end{array}$ & $\begin{array}{l}257 \\
16.1\end{array}$ & $\begin{array}{l}71 \\
4.4\end{array}$ & $\begin{array}{l}46 \\
2.9\end{array}$ & $\begin{array}{l}251 \\
15.7\end{array}$ & $\begin{array}{l}147 \\
9.2\end{array}$ \\
\hline 2008/09 & $\begin{array}{l}308 \\
19.4\end{array}$ & $\begin{array}{l}188 \\
11.8\end{array}$ & $\begin{array}{l}42 \\
2.6\end{array}$ & $\begin{array}{l}345 \\
21.7\end{array}$ & $\begin{array}{l}86 \\
5.4\end{array}$ & $\begin{array}{l}76 \\
4.8\end{array}$ & $\begin{array}{l}317 \\
19.9\end{array}$ & $\begin{array}{l}168 \\
10.6\end{array}$ \\
\hline 2009/10 & $\begin{array}{l}273 \\
17.2\end{array}$ & $\begin{array}{l}185 \\
11.6\end{array}$ & $\begin{array}{l}29 \\
1.8\end{array}$ & $\begin{array}{l}322 \\
20.2\end{array}$ & $\begin{array}{l}97 \\
6.1\end{array}$ & $\begin{array}{l}58 \\
3.6\end{array}$ & $\begin{array}{l}327 \\
20.6\end{array}$ & $\begin{array}{l}167 \\
10.5\end{array}$ \\
\hline $2003 / 04-2009 / 10$ & $\begin{array}{l}2320 \\
20.7\end{array}$ & $\begin{array}{l}1268 \\
11.3\end{array}$ & $\begin{array}{l}241 \\
2.2\end{array}$ & $\begin{array}{l}2313 \\
20.7\end{array}$ & $\begin{array}{l}496 \\
4.4\end{array}$ & $\begin{array}{l}367 \\
3.3\end{array}$ & $\begin{array}{l}1914 \\
17.1\end{array}$ & $\begin{array}{l}1188 \\
10.6\end{array}$ \\
\hline \multicolumn{9}{|l|}{ Females } \\
\hline 2003/04 & $\begin{array}{l}373 \\
24.4\end{array}$ & $\begin{array}{l}121 \\
7.9\end{array}$ & $\begin{array}{l}22 \\
1.4\end{array}$ & $\begin{array}{l}241 \\
15.8\end{array}$ & $\begin{array}{l}66 \\
4.3\end{array}$ & $\begin{array}{l}56 \\
3.7\end{array}$ & $\begin{array}{l}180 \\
11.8\end{array}$ & $\begin{array}{l}145 \\
9.5\end{array}$ \\
\hline 2004/05 & $\begin{array}{l}444 \\
29.0\end{array}$ & $\begin{array}{l}121 \\
7.9\end{array}$ & $\begin{array}{l}15 \\
1.0\end{array}$ & $\begin{array}{l}226 \\
14.8\end{array}$ & $\begin{array}{l}70 \\
4.6\end{array}$ & $\begin{array}{l}41 \\
2.7\end{array}$ & $\begin{array}{l}187 \\
12.2\end{array}$ & $\begin{array}{l}137 \\
9.0\end{array}$ \\
\hline 2005/06 & $\begin{array}{l}391 \\
25.6\end{array}$ & $\begin{array}{l}120 \\
7.9\end{array}$ & $\begin{array}{l}27 \\
1.8\end{array}$ & $\begin{array}{l}209 \\
13.7\end{array}$ & $\begin{array}{l}50 \\
3.3\end{array}$ & $\begin{array}{l}58 \\
3.8\end{array}$ & $\begin{array}{l}220 \\
14.4\end{array}$ & $\begin{array}{l}137 \\
9.0\end{array}$ \\
\hline 2006/07 & $\begin{array}{l}353 \\
23.1\end{array}$ & $\begin{array}{l}121 \\
7.9\end{array}$ & $\begin{array}{l}16 \\
1.0\end{array}$ & $\begin{array}{l}228 \\
14.9\end{array}$ & $\begin{array}{l}74 \\
4.9\end{array}$ & $\begin{array}{l}44 \\
2.9\end{array}$ & $\begin{array}{l}175 \\
11.5\end{array}$ & $\begin{array}{l}106 \\
6.9\end{array}$ \\
\hline 2007/08 & $\begin{array}{l}362 \\
23.8\end{array}$ & $\begin{array}{l}100 \\
6.6\end{array}$ & $\begin{array}{l}23 \\
1.5\end{array}$ & $\begin{array}{l}235 \\
15.5\end{array}$ & $\begin{array}{l}45 \\
3.0\end{array}$ & $\begin{array}{l}40 \\
2.6\end{array}$ & $\begin{array}{l}170 \\
11.2\end{array}$ & $\begin{array}{l}125 \\
8.2\end{array}$ \\
\hline 2008/09 & $\begin{array}{l}373 \\
24.6\end{array}$ & $\begin{array}{l}111 \\
7.3\end{array}$ & $\begin{array}{l}24 \\
1.6\end{array}$ & $\begin{array}{l}257 \\
17.0\end{array}$ & $\begin{array}{l}78 \\
5.1\end{array}$ & $\begin{array}{l}47 \\
3.1\end{array}$ & $\begin{array}{l}262 \\
17.3\end{array}$ & $\begin{array}{l}164 \\
10.8\end{array}$ \\
\hline
\end{tabular}


Table 4 nTBI episodes of care (n and rate per 100,000 children and youth aged 19 years and under) in Ontario between fiscal years 2003/04 and 2009/10 by type of nTBI and sex (Continued)

\begin{tabular}{lllllllll}
\hline $2009 / 10$ & 352 & 120 & 13 & 313 & 84 & 40 & 258 & 139 \\
& 23.2 & 7.9 & 0.9 & 20.6 & 5.5 & 2.6 & 3.0 & 9.2 \\
$2003 / 04-2009 / 10$ & 2648 & 814 & 140 & 1709 & 467 & 326 & 1452 & 953 \\
& 24.8 & 7.6 & 1.3 & 16.0 & 4.4 & 3.1 & 13.6 & 8.9 \\
\hline
\end{tabular}

half of the patients with a toxic effect of substance stayed in acute care for less than 2 days. However, $46 \%$ of cases with vascular insult stayed in acute care for 12 days or longer. Almost $60 \%$ of cases with vascular insult stayed in the intensive care unit for at least one day, compared to approximately a quarter of cases with encephalitis and meningitis. Overall, there was a small percentage of deaths in acute care, however, $19 \%$ of all patients with anoxic brain injury died in acute care (Table 3 ).

\section{Discussion}

This study is the first study, to the best of our knowledge, providing a comprehensive overview of the burden of pediatric nTBI on healthcare services. Consistent with the trends seen in the overall nTBI population in Ontario [9], the number of nTBI episodes of care among children and youth increased from 2003/04 to 2009/10. However, differences were observed in discharge patterns. Of note is that the majority of children and youth were discharged home post-acute care, of which $9 \%$ were discharged home with support services. This differs from findings on the overall nTBI population in Ontario, where less than $40 \%$ were discharged home and $20 \%$ died in acute care $[3,9]$. This finding on discharge home is in line with studies that indicate a preference to discharge children and youth home post injury [41]. Given these differences in the healthcare use of the pediatric population compared to the general population, a specific focus on the health service use of this pediatric population is needed.

The pediatric nTBI population also differs from the TBI population; although the rate of nTBI episodes of care in this study was not as high as the rates reported for the TBI population, which ranged from 125 to 1,337 per $100,000[34,42,43]$, the health service use of the nTBI population is just as high as the TBI population. For example, it was reported that the average LOS for a TBI related hospitalization in Canada was 5 days [44]; this paper showed that that the average LOS for a nTBI related hospitalization was approximately 13 days. Further, death in acute care for the TBI population was reported to be $9 \%$ in Canada [44]. However, among those with anoxic brain injury, up to $19 \%$ died in acute care. Although nTBI is not as common as TBI, this population puts an increased burden and demand on the healthcare system. Moreover, there is relatively little data suggesting how we can effectively and appropriately allocate the resources and support for these patients. Given that patients with TBI and nTBI are often treated in similar settings $[13,14]$ and that they use almost triple the amount of services, data pertaining specifically to the nTBI population is crucial.

Equally important is an understanding of the profile and use of healthcare services associated with each subtype of nTBI. For example, despite an overall increasing rate of nTBI episodes of care, differences were observed between types of nTBI, which may be due to the improvement in diagnosis or treatment, prevention, or coding practices. For example, there was an overall decrease in the rate of toxic effect of substances episodes of care, which may be attributed to increased prevention efforts against hazardous drinking, particularly in adolescents. Conversely, the slight increase in brain tumour episodes of care may reflect better detection of brain tumours

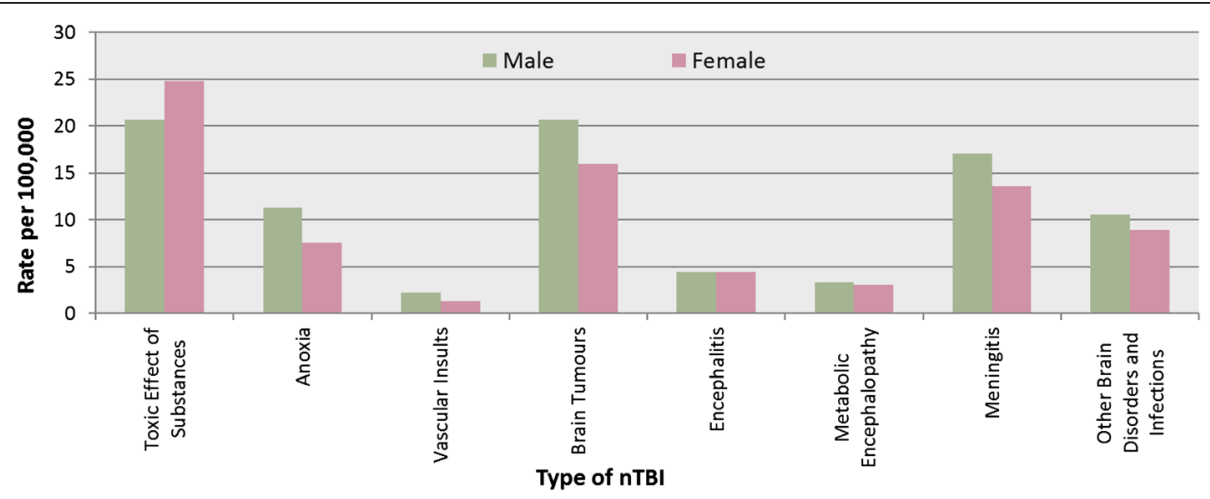

Fig. 3 nTBI episodes of care by type of nTBI and sex 


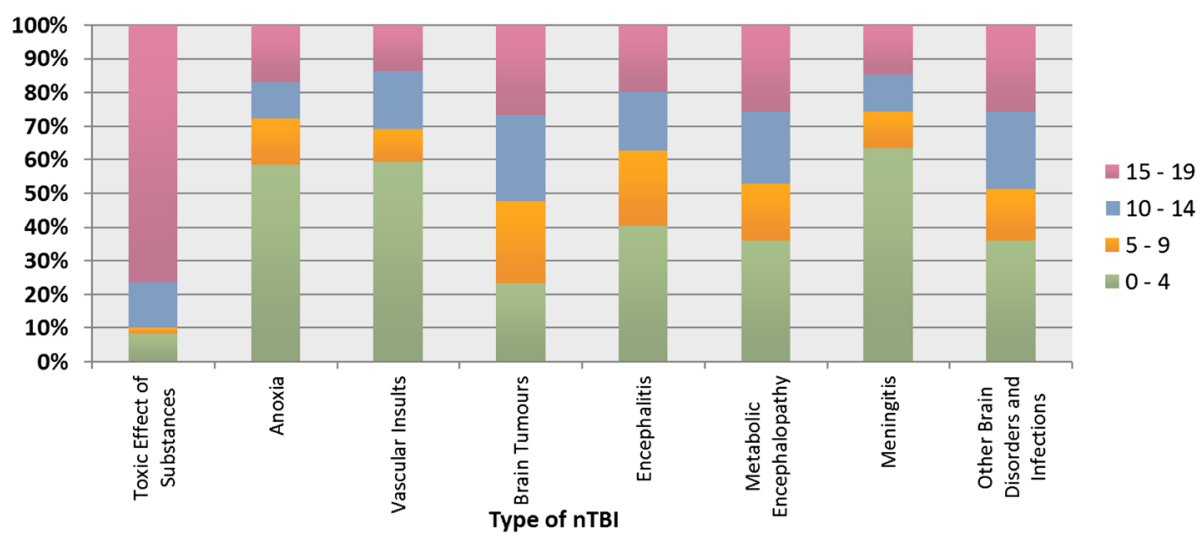

Fig. 4 Distribution of age by type of nTBI

while the increase in encephalitis may reflect trend towards a diagnosis/coding of "unspecified" or "unknown" cause of encephalitis [45-48], a diagnosis that is captured in this study. These preliminary data on subtypes of nTBI highlight the importance of stratifying the nTBI population by subtype. Also, consistent with studies on anoxic brain injury among the pediatric population [19, 20], nearly one in five patients died in acute care. Data stratified by cause of anoxic brain injury can inform the prevention of anoxic brain injuries and preparation of healthcare for this population, as it has been reported that outcomes of anoxic brain injury from a cardiac arrest vs. near drowning are significantly different [20]. Furthermore, the differences in discharge destinations across types of nTBI reflect the complexities and diversity of this group. While very few patients with a diagnosis of toxic effect of substances are discharged home with support services, more than a fifth are transferred to another inpatient facility. Centres that receive these patients, including addiction treatment centres, should be aware of and consider the effects of a brain injury on these patients when assessing treatment options. Finally, the distribution of age group across each subtype of nTBI is also varied, presenting opportunities for targeted interventions by age. These data by subtype of nTBI provide the foundation needed for further in-depth research on each type of nTBI to inform targeted resource allocation and healthcare planning.

Limitations associated with this study include a lack of consistent, agreed upon case definition for nTBI. The importance of this is highlighted in a comparison of data presented by Wong and colleagues in 2001 on nontraumatic coma. Despite similar goals on informing healthcare resources for this population, the method used to identify patients were different, resulting in higher rates of nTBI episodes of care $(82.3$ per 100,000$)$ reported in this study compared to data presented by Wong and colleagues (30.8 per 100,000) [49]. Specifically, Wong and colleagues restricted participants to only those with "significantly depression of conscious level as defined by the Glasgow Coma Score of 12" and, among those under the age of 5 years, at least $6 \mathrm{~h}$ of unconsciousness. Conversely, this paper identified nTBI using ICD-10 codes and the codes included in this study likely captured patients with a less severe nTBI. Limitations related to the data source are also recognized. First, the DAD only captures individuals that are admitted to an acute care setting and thus, deaths that occur outside of the hospital are missed, which is considered a limitation in neurotrauma research [50]. Second, the use of all 10 diagnosis fields in the NACRS and 25 diagnosis fields in the DAD resulted in counting of all multiple nTBI diagnoses, as a patient may have more than one type of nTBI. This is an important methodological issue to consider for future studies involving statistical analyses between and/or across each type of nTBI. Third, the use of only the DAD and the NACRS to identify cases of brain tumours may result in underestimates, as brain tumours may not be readily identified or captured in the ED and/ or acute care setting. The inclusion of the Ontario Cancer Registry [51], which captures information on all newly diagnosed cases of invasive neoplasia, may result in a higher number of brain tumour cases captured. As such, it is acknowledged that estimates of brain tumours in this study are likely underestimates. Nonetheless, effort to capture all potential cases that may present in the emergency department or acute care was made by using episodes of care to assess the burden of healthcare services. For example, toxic effects of substances are likely to present in the emergency department, however, this diagnosis may not be made in the acute care setting. As such, the linkage of the DAD to the NACRS helped ensure that cases that are not coded as 'toxic effects of substances' in the DAD were captured in the NACRS and that double counting did not occur. The data sources used in this study are also population based and Ontario has publicly funded healthcare. Therefore, data 
presented here are less likely to have been influenced by access supplemental health insurance. Finally, it is acknowledged that the Charlson Comorbidity Index may not be ideal for comorbidity information in the children and youth population. However, this was chosen to provide consistency for comparison with published population based data on the adult and older adult $\mathrm{ABI}$ populations in Ontario, Canada $[3,4,7,8]$. It was also used to assess the severity of comorbid health conditions, which has been used previously in research looking at the pediatric population [39]. Future studies should consider exploring comorbidities through ICD10 Chapter Headings [52] or the John Hopkins Aggregated Diagnostic Grouping (ADG) [53].

\section{Conclusions}

To the best of our knowledge, this is the first populationbased study to provide an overview of children and youth with nTBI in Ontario, Canada. Findings from this study provide a foundation for further in-depth research on nTBI and its subtypes that are critical for resource allocation and support for children and youth with nTBI. The availability of accurate and timely information on nTBI is crucial for the planning of healthcare services, resource allocation, and prevention. Children and youth are at a critical developmental period of their lives in which a nTBI can result in negative and lasting consequences. Some types of nTBI identified in this study are preventable; a focus on nTBI and in-depth research on each subtypes of nTBI is encouraged.

\section{Abbreviations \\ $\mathrm{ABI}$, acquired brain injury; CCC, complex continuing care: $\mathrm{CIHI}$, Canadian Institute for Health Information; DAD, Discharge Abstract Database; ICD-10, International Classification of Diseases Version 10; LOS, length of stay; LTC, long-term care; NACRS, National Ambulatory Care Reporting System; nTBI. non-traumatic brain injury; TBI, traumatic brain injury}

\section{Funding}

VC received support from the Jane Gillett Pediatric ABI Studentship through the Ontario Neurotrauma Foundation, the Doctoral Research Award from the Canadian Institutes of Health Research (CIHR) and Pediatric Oncology Group of Ontario, and a Brain Canada-CIBC Brain Cancer Training Award from Brain Canada and CIBC. AC received support through a CIHR Research Chair in Gender, Work and Health (\#CGW-126580). The authors have no conflicts of interest.

\section{Availability of data and materials}

We would like to thank the Ontario Ministry of Health and Long-Term Care for providing us with the data. The views expressed are those of the Principal Researchers and do not necessarily reflect those of the Ontario or the Ministry. Data sharing is currently unavailable, as data can only be released in a manner consistent with the Ontario Privacy Commissioner approved by the Ontario Ministry of Health and Long-Term Care policies and procedures.

\section{Authors' contribution}

VC and AC conceptualized the study. VC formulated the methods for statistical analysis and carried out the analysis using SAS software. VC drafted the paper, conducted the literature review, and interpreted the results that formulated the foundation of the paper. VC, AC, JP, MK, and RM all had significant input into the editing process of this paper and additional interpretation of results. All authors approved the final manuscript.

\section{Competing interests}

The authors declare that they have no competing interests.

\section{Consent for publication}

Consent from patients is not possible as the data sources for this study are de-identified healthcare administrative databases.

\section{Ethics approval and consent to participate}

Ethics approval for this study was obtained from the Toronto Rehabilitation Institute, University Health Network Research Ethics Board. Informed consent from patients is not possible as the data sources for this study are de-identified healthcare administrative databases.

\section{Author details}

${ }^{1}$ Toronto Rehabilitation Institute, University Health Network, 550 University Avenue, Toronto, ON M5G 2A2, Canada. ${ }^{2}$ Rehabilitation Sciences Institute, University of Toronto, 500 University Avenue, Toronto, ON M5G 1 V7, Canada. ${ }^{3}$ Pediatric Oncology Group of Ontario, 480 University Avenue, Toronto, ON M5G 1 V2, Canada. "Dalla Lana School of Public Health, University of Toronto, 155 College Street, Toronto, ON M5T 3 M7, Canada. ${ }^{5}$ Holland Bloorview Kids Rehabilitation Hospital, 150 Kilgour Road, Toronto, ON M5G 1R8, Canada. ${ }^{6}$ Centre for Addiction and Mental Health, 33 Russell Street, Toronto, ON M5S 3M1, Canada.

Received: 7 November 2015 Accepted: 1 July 2016

Published online: 20 July 2016

\section{References}

1. CARF International. CARF-CCAC Standards Manual 2015. Tucson, Arizona, USA

2. Giustini A, Pistarini C, Pisoni C. Traumatic and nontraumatic brain injury. Handb Clin Neurol. 2013:110:401-9.

3. Chan $V$ et al. Older adults with acquired brain injury: a population based study. BMC Geriatr. 2013:13:97.

4. Chan $V$ et al. Older adults with acquired brain injury: outcomes after inpatient rehabilitation. Can J Aging. 2013;32(3):278-86.

5. Chen A, Zagorski B, Chan V, Parsons D, Vander Laan R, Colantonio A. Acute care alternate level of care days due to delayed discharge for traumatic and non-traumatic brain injuries. Healthcare Policy. 2013;7(4):41-55.

6. Chen $\mathrm{A}$ et al. Direct cost associated with acquired brain injury in Ontario. BMC Neurol. 2012;12:76

7. Chen $A$ et al. Factors associated with living setting at discharge from inpatient rehabilitation after acquired brain injury in Ontario, Canada. J Rehabil Med. 2014;46(2):144-52.

8. Chen AY et al. Factors associated with discharge destination from acute care after acquired brain injury in Ontario, Canada. BMC Neurol. 2012;12:16

9. Colantonio A, Chan V, Zagorski B, Parsons D. Ontario acquired brain injury (ABI) dataset project phase III: Highlights, numbers of episodes of care and causes of brain injury. Toronto: Acquired Brain Injury Research Lab; 2012.

10. Colantonio A, Chan V, Zagorski B, Parsons D. Ontario Acquired Brain Injury (ABI) Dataset Project Phase III: Highlights: Alternate level of care (ALC) days, length of stay, and discharge disposition of ABI patients. Toronto, Ontario, Canada: Acquired Brain Injury Research Lab; 2012.

11. Cullen NK, Crescini C, Bayley MT. Rehabilitation outcomes after anoxic brain injury: a case-controlled comparison with traumatic brain injury. PMR. 2009; 1(12):1069-76

12. Cullen NK, Park YG, Bayley MT. Functional recovery following traumatic vs non-traumatic brain injury: a case-controlled study. Brain Inj. 2008;22(13-14): 1013-20.

13. Brain Injury Rehabiltiation Program. 2015. Available from: http://rusk.med. nyu.edu/brain-injury-rehabilitation-program. Accessed 7 July 2015.

14. Brain Injury Rehab including Concussions. 2015. Available from: http:// hollandbloorview.ca/programsandservices/ProgramsServicesAZ/ Braininjuryrehab. Accessed 7 July 2015.

15. Forsyth R, Kirkham F. Predicting outcome after childhood brain injury. CMAJ 2012;184(11):1257-64.

16. Canadian Institute for Health Information. The burden of neurological diseases, disorders, and injuries in Canada. Ottawa: $\mathrm{ClHI} ; 2007$

17. Canadian Brain Tumour Registry 2015. Available from: http://www. braintumour.ca/4475/brain-tumour-registry. Accessed 7 July 2015.

18. Greenberg ML, Barnett H. Atlas of Childhood Cancer in Ontario, 1985-2004. Toronto: Pediatric Oncology Group of Ontario; 2015. 
19. Donoghue AJ et al. Out-of-hospital pediatric cardiac arrest: an epidemiologic review and assessment of current knowledge. Ann Emerg Med. 2005;46(6):512-22.

20. Johnson AR, DeMatt E, Salorio CF. Predictors of outcome following acquired brain injury in children. Dev Disabil Res Rev. 2009;15(2):124-32.

21. Holmquist L, Russo CA, Elixhauser A. Meningitis-Related Hospitalizations in the United States, 2006: Statistical Brief \#57, in Healthcare Cost and Utilization Project (HCUP) Statistical Briefs. Rockville (MD): Agency for Healthcare Research and Quality; 2006.

22. Hviid A, Melbye M. The epidemiology of viral meningitis hospitalization in childhood. Epidemiology. 2007;18(6):695-701.

23. Kneen $\mathrm{R}$ et al. Management of suspected viral encephalitis in children Association of British Neurologists and British Paediatric Allergy, Immunology and Infection Group national guidelines. J Infect. 2012;64(5):449-77.

24. Weingarten L, Enarson P, Klassen T. Encephalitis. Pediatr Emerg Care. 2013; 29(2):235-41. quiz 242-4.

25. Emergency and Ambulatory Care. 2015. Available from: https://www.cihi.ca/ en/types-of-care/hospital-care/emergency-and-ambulatory-care. Accessed 7 July 2015.

26. Acute Care. 2015. Available from: https://www.cihi.ca/en/types-of-care/ hospital-care/acute-care. Accessed 7 July 2015.

27. Juurlink D, Preyra C, Croxford R, Chong A, Austin P, Tu J, Laupacis A. Canadian Institute for Health Information Discharge Abstract Database: A Validation Study. Toronto, Ontario, Canada: Institute for Clinical Evaluative Sciences; 2006.

28. Colantonio A, Parsons D, Vander Laan R, Zagorski B. ABI Dataset Pilot Project Phase 1 Report. Toronto: Ontario Neurotrauma Foundation; 2009.

29. Centers for Medicare \& Medicaid Serices. The Inpatient Rehabiltiation Facility Patient Assessment Instrument (IRF-PAI) Training Manual. UB Foundation Activities, Inc; 2012.

30. Background on the National Rehabilitation Reporting System. Available from: http://www.cihi.ca/CIHI-ext-portal/internet/en/document/types+of+care/ hospital+care/rehabilitation/services_nrs_intro. Accessed 7 July 2015

31. About our programs. Available from: http://www.uhn.ca/TorontoRehab. Accessed 18 May 2016

32. Departments and programs. Available from: http://rusk.med.nyu.edu/ patients-families/departments-programs. Accessed 18 May 2016.

33. Evidence based review of rehabilitation of moderate to severe acquired brain injuries. Defining acquired brain injury. . 2011. Available from: http:// www.abiebr.com/module/1-introduction-and-methodology. Accessed 18 May 2016.

34. Chan $V$, Thurairajah $P$, Colantonio A. Defining pediatric traumatic brain injury using International Classification of Diseases Version 10 Codes: a systematic review. BMC Neurol. 2015;15:7.

35. Population by sex and age group 2015. Available from: http://www.statcan.gc ca/tables-tableaux/sum-som/101/cst01/demo10a-eng.htm. Accessed 10 July 2015.

36. Ahmad OB, Boschi-Pinto C, Lopez AD, Murray CL, Lozano R, Inoue M. Age standardization of rates: A new WHO standard. World Health Organization; 2001. http://www.who.int/healthinfo/paper31.pdf.

37. Charlson ME et al. A new method of classifying prognostic comorbidity in longitudinal studies: development and validation. J Chronic Dis. 1987;40(5): 373-83.

38. Sundararajan $V$ et al. New ICD-10 version of the Charlson comorbidity index predicted in-hospital mortality. J Clin Epidemiol. 2004;57(12):1288-94.

39. Charlson $\mathrm{M}$ et al. The Charlson comorbidity index can be used prospectively to identify patients who will incur high future costs. PLoS One. 2014;9(12): e112479.

40. Colantonio A, Saverino C, Zagorski B, Swaine B, Lewko J, Jaglal S, Vernich L. Hospitalizations and emergency department visits for TBI in Ontario Canadian. J Neurol Sci. 2010;37:783-90.

41. DeMatteo CA et al. Exploring postinjury living environments for children and youth with acquired brain injury. Arch Phys Med Rehabil. 2008;89(9):1803-10.

42. Faul M, Xu L, Wald MM, Coronado VG. Trauamtic brian injury in the United States: Emergency department visits, hospitalziations, and deaths 2002 2006. Centers for Disease Control and Prevention, National Center for Injury Prevention and Control Atlanta; 2010.

43. Feigin $\mathrm{VL}$ et al. Incidence of traumatic brain injury in New Zealand: a population-based study. Lancet Neurol. 2013;12(1):53-64.

44. Canadian Institute for Health Information. Head injuries in Canada: A decade of change (1994-1995 to 2003-2004). Ottawa: CIHI; 2006.
45. Barbadoro $\mathbf{P}$ et al. Trend of hospital utilization for encephalitis. Epidemiol Infect. 2012;140(4):753-64

46. Britton PN et al. Acute encephalitis in children: Progress and priorities from an Australasian perspective. J Paediatr Child Health. 2015:51(2):147-58.

47. Koskiniemi $M$ et al. Infections of the central nervous system of suspected viral origin: a collaborative study from Finland. J Neurovirol. 2001;7(5):400-8

48. Olsen SJ et al. Infectious causes of encephalitis and meningoencephalitis in Thailand, 2003-2005. Emerg Infect Dis. 2015;21(2):280-9.

49. Wong CP et al. Incidence, aetiology, and outcome of non-traumatic coma: a population based study. Arch Dis Child. 2001;84(3):193-9.

50. Parsons D, Colantonio A, Mohan M. The utility of administrative data for neurotrauma surveillance and prevention in Ontario, Canada. BMC Res Notes. 2012;5:584.

51. Ontario Cancer Registry 2015. Available from: https://www.cancercare.on.ca/ ocs/csurv/ocr/. Accessed 8 July 2015.

52. Saverino C, et al. Re-hospitalization after Traumatic Brain Injury: A Population Based Study. Arch Phys Med Rehabil. 2016;97(Suppl 2):S19-S25.

53. The Johns Hopkins ACG System. Baltimore, MD, USA: Johns Hopkins Bloomberg School of Public Health; 2009.

\section{Submit your next manuscript to BioMed Central and we will help you at every step:}

- We accept pre-submission inquiries

- Our selector tool helps you to find the most relevant journal

- We provide round the clock customer support

- Convenient online submission

- Thorough peer review

- Inclusion in PubMed and all major indexing services

- Maximum visibility for your research

Submit your manuscript at www.biomedcentral.com/submit

) Biomed Central 